\title{
Therapeutic Efficacy and Safety of Invasive Pulsed-Type Bipolar Alternating Current Radiofrequency on Melasma and Rebound Hyperpigmentation
}

Hyoung Moon Kim

Min Ji Lee

Miaero Clinic, Seoul, Korea
Received June 19, 2017

Accepted June 21, 2017

\section{Correspondence}

Hyoung Moon Kim

Miaero Clinic, 164 Gwanak-ro, Gwanak-gu, Seoul 08788, Korea

Tel.: +82-2-871-7711

Fax: +82-2-876-7791

E-mail: drmac12ame.com

(C) Korean Society for Laser Medicine and Surgery

(c) This is an open access article distributed under the terms of the Creative Commons Attribution NonCommercial License (http://creativecommons.org/ licenses/by-nc/4.0) which permits unrestricted noncommercial use, distribution, and reproduction in any medium, provided the original work is properly cited.

\begin{abstract}
Background and Objectives
Laser treatment for melasma is typically associated with various complications, especially rebound hyperpigmentation (RH) and mottled hypopigmentation. The purpose of this case review was to analyze the clinical efficacy and safety of using an uinvasive pulsed-type bipolar alternating current radiofrequency (IBPRF) for treating melasma with or without $\mathrm{RH}$.
\end{abstract}

\section{Materials and Methods}

Clinical improvement in the appearances of melasma and $\mathrm{RH}$ was analyzed in 142 Korean patients $(4$ men and 138 women, with a mean age of $37.3 \pm 11$ years), who had undergone IBPRF treatment for melasma. IBPRF was delivered at a frequency of $2 \mathrm{MHz}$ and with non-insulated, penetrating microneedles over five to 10 treatment sessions at 2- to 3-week intervals.

\section{Results}

In total, 124 patients had melasma, and among them,18 patients had melasma with RH. To treat melasma, IBPRF alone was administered to 112 subjects, IBPRF plus a Q-switched Q-switched neodymium-yttriumaluminum-garnet (Nd:YAG) laser in 22 patients, IBPRF plus intralesional tranexamic acid injection in five patients, and IBPRF plus bleaching cream in three patients. Patients reported subjective satisfaction with the appearance of their melasma lesions after seven to eight treatment sessions, regardless of whether they underwent treatment with pulsed RF alone or combination treatment. After treatment, no significant complications, particularly $\mathrm{RH}$ or mottled hypopigmentation, were recorded.

\section{Conclusion}

No severe complications related to IBPRF treatment were recorded, and patients reported satisfaction in the appearance of their lesions after seven to eight treatment sessions. This suggests that IBPRF may be effective and safe for treating melasma and $\mathrm{RH}$.

\section{Key words}

Invasive pulsed-type bipolar radiofrequency; Melasma; Postinflammatory hyperpigmentation; Rebound hyperpigmentation 


\section{INTRODUCTION}

Melasma is a commonly acquired hyperpigmentary disorder characterized by light to dark brown macules and patches occurring along the sun-exposed areas of the face and neck. 'The treatment of melasma remains a challenge. Numerous treatment options, including topical agents, chemical peels, and laser treatments, have been applied for treating melasma. ${ }^{1} \mathrm{Q}$-switched pigmentary lasers have not been recommended for melasma treatment due to a high incidence of postinflammatory hyperpigmentation (PIH) and unpredictable efficacy. Intense pulsed light has shown some efficacy in the treatment of melasma, although the risk of PIH still remains high. ${ }^{2}$ Fractional laser treatments have also been reported to improve melasma. However, a recent report showed that the therapeutic efficacy of fractional lasers did not differ from that of conventional therapies. ${ }^{3}$

Recently, a 1064-nm Q-switched neodymium-yttriumaluminum-garnet (Nd:YAG) laser with low energy was introduced for the treatment of melasma in Asian patients, with favorable results. ${ }^{4-6}$ Patients experienced minimal downtime without crust formation and significant erythema after laser treatment. Notwithstanding, the possibility of rebound hyperpigmentation (RH) and punctate leukoderma have since been found to be the major concerns for this treatment. ${ }^{7.8}$

Studies have shown that the skin behaves like a semiconductor. ${ }^{9}$ When emitted on the skin, radiofrequency (RF) elicits epidermal and dermal heating. Electrical conductivity is an important factor affecting RF conduction, and research has shown that tissue hydration and temperature are important conductivity factors. ${ }^{10,11}$ When RF current is applied to the skin, an electrobiological change is elicited therein. At a minimal pulse duration, however, little to no change in the epidermis and dermis can be achieved: "pulsed" describes intermittent durations during which the laser is switched on and off. Nonetheless, with time, various changes in the skin have been recorded..$^{10}$ The purpose of this study was to evaluate the clinical efficacy and safety of treating melasma and $\mathrm{RH}$ with invasive pulsed-type bipolar alternating current radiofrequency (IBPRF) with non-insulated needles.

\section{MATERIALS AND METHODS}

\section{Patients and radiofrequency treatment}

The present study was conducted as a review of medical records for 142 patients who underwent treatment with IBPRF for melasma with or without $\mathrm{RH}$. The patients were clinically diagnosed with melasma. After obtaining written informed consent, melasma lesions with or without $\mathrm{RH}$ were treated with pulsed-type RF in a bipolar mode, at a frequency of $2 \mathrm{MHz}$, and with a disposable tip comprising 25 non-insulated, penetrating microneedles in a uniform $5 \times 5$ array (SYLFIRM ${ }^{\top M}$; Viol, Kyunggi, Korea) (Fig. 1). At baseline and one month after the final treatment, photographs were taken under normal and ultraviolet light exposures with an imaging tool to evaluate improvement in the appearance of treated lesions.

All procedures using IBPRF were performed after applying local anesthesia with topical EMLA cream leutectic mixture of 2.5\% lidocaine $\mathrm{HCl}$ and 2.5\% prilocaine; AstraZeneca AB, Södertälje, Sweden). Parameters for IBPRF were set at level III and a $1.5-\mathrm{mm}$ penetration depth over a single session for treatment along the face and at level II and a 1-mm depth for treatment along the forehead. Overall, five to 10 treatment sessions were proceeded at intervals of 2 to 3 weeks. Immediately after treatment, a hydration mask pack was applied. Neither systemic nor topical corticosteroids and antibiotics were prescribed prophylactically to most of the patients. The patients were recommended to apply a broad-spectrum sunscreen and avoid excessive sun exposure during the course of laser treatments.

\section{Combined treatment}

Among the 142 patients treated with IBPRF, a combination of IBPRF plus a Q-switched Nd:YAG laser was applied in 22, IBPRF plus intralesional tranexamic acid injection was administered in five, and IBPRF plus bleaching cream was applied in three. In each of the 22 patients treated with a Q-switched Nd:YAG laser, the whole face was treated with a 1,064-nm Q-switched single pulse $\mathrm{Nd}: Y A G$ laser with the settings of $1.0-1.2 \mathrm{~J} / \mathrm{cm}^{2}$, a pulse duration of 5-10-nsec, and a 7-mm spot size. A total of

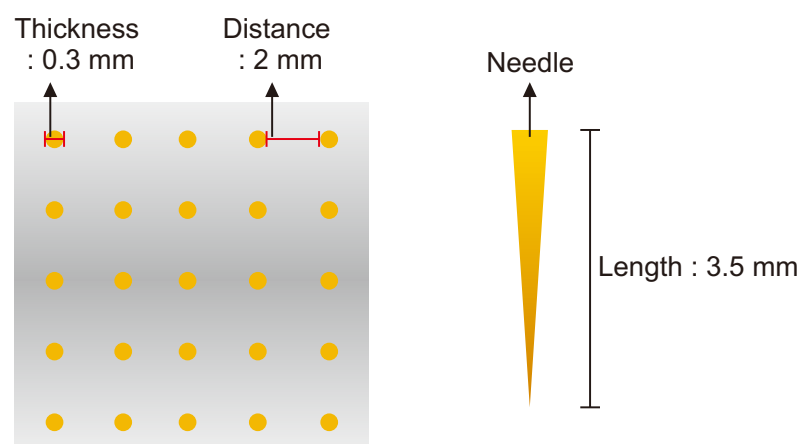

Fig. 1. Schematic illustration presenting the characteristics of microneedle electrodes in a disposable tip. 
2,000 to 2,500 shots were delivered on the entire face. The IBPRF treatments were performed immediately thereafter. Intralesional tranexamic acid injection therapy was delivered immediately after IBPRF treatment.

\section{RESULTS}

Over 1 year, a total of 142 patients underwent IBPRF treatment for melasma with or without $\mathrm{RH}$. Of these, $97 \%$ were female and $3 \%$ were male. Fifteen patients reported having had melasma for less than 1 year, 64 patients stated between 1 to 5 years, and 53 patients reported having melasma for longer than 5 years but less than 10 years. There were 10 patients who had melasma for more than 10 years. Regarding melasma types, 103 patients presented with melasma of centrofacial type, while the other
27 patients exhibited melasma of malar type.

Among all patients, 124 reported that they had not previously undergone treatment for melasma, while 18 had. Of these 18 patients, laser toning was most commonly used for melasma treatment, followed by treatment with intense pulsed light or whitening ointment. Some had also received combination treatment with a fractional laser, tranexamic acid injection, and polydeoxyribonucleotide injections. All 18 patients had undergone basic treatment with laser toning combined with other treatment.

For melasma treatment, IBPRF alone was administered in 112 subjects. A combination of IBPRF plus a Qswitched Nd:YAG laser was applied in 22, IBPRF plus intralesional tranexamic acid injection was administered in five, and IBPRF plus bleaching cream was applied in three. Surprisingly, patients generally reported subjective
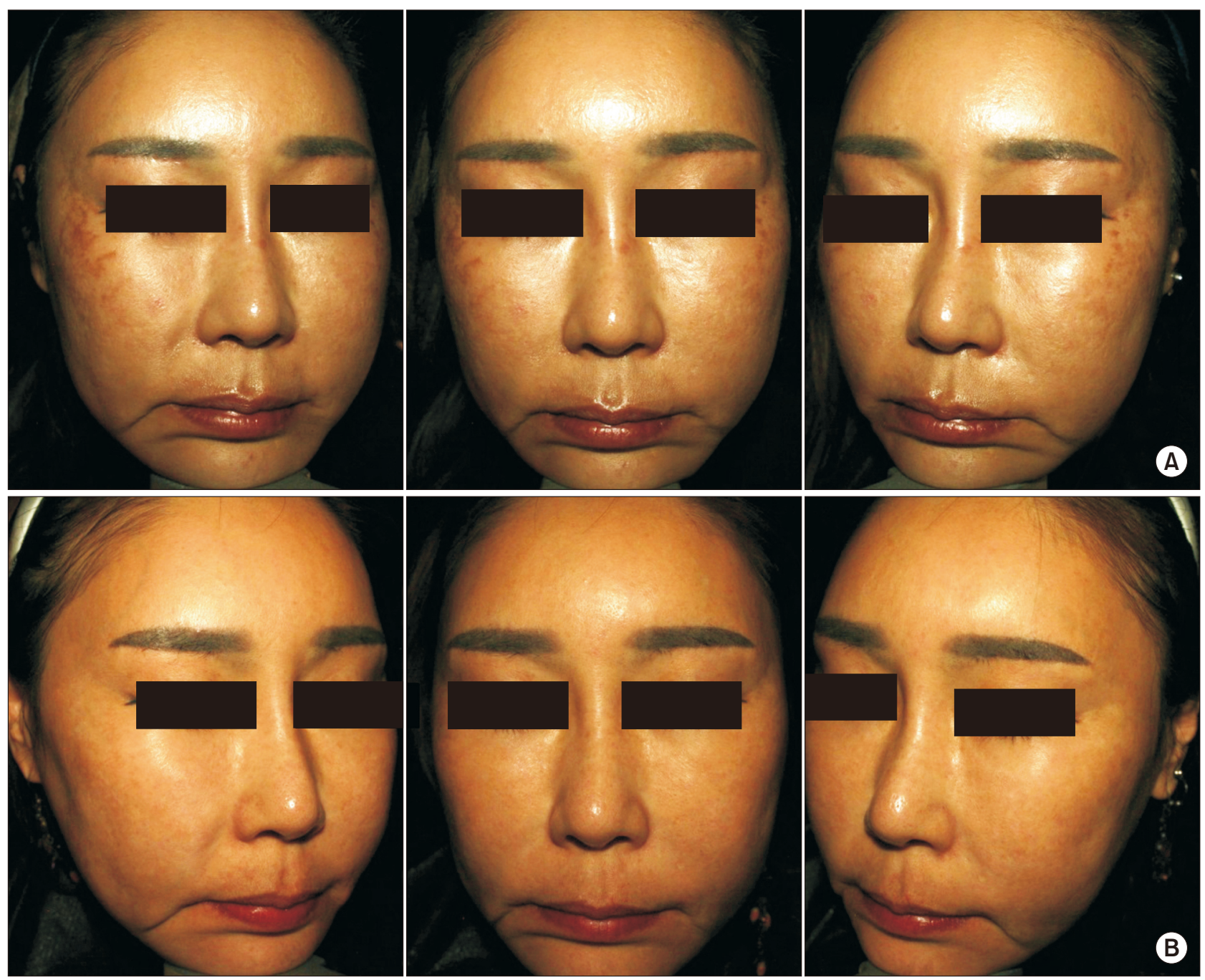

Fig. 2. Representative photographs of a 46-year-old female patient with recurrent melasma. (A) Baseline and (B) one month after ten sessions of invasive pulsed-type bipolar alternating current radiofrequency (IBPRF) monotherapy. 
satisfaction with the appearance of their melasma lesions after seven to eight treatment sessions, regardless of whether they underwent treatment with pulsed RF alone or combination treatment (Figs. 2-4). Furthermore, most of the patients exhibited overall clinical improvement in skin texture and tone.

During the course of IBPRF treatment and follow up, no significant complications, particularly RH or mottled hypopigmentation, were recorded. The most common complication was induration at the treatment area due to excess exposure to radiofrequency energy; it disappeared within 2 to 3 days of applying steroid ointment. Moreover, no other side effects, including bruise, bleeding, oozing, prolonged erythema, folliculitis, acneiform eruption, and scarring, were encountered.

\section{DISCUSSION}

In the present study, we aimed to determine the clinical efficacy and safety of treating melasma and $\mathrm{PIH}$ with pulsed RF treatment. In 142 patients, IBPRF treatment was delivered at a frequency of $2 \mathrm{MHz}$ and with noninsulated, penetrating microneedles over five to 10 treatment sessions at 2- to 3-week intervals. After treatment, no significant complications, particularly RH or mottled hypopigmentation, were recorded.

Generally, melanin is not believed to be influenced by RF treatment. We, however, suggest that morphological or functional melanin structures are directly changed upon RF irradiation of melasma lesions. The electrical properties of melanin have been shown to be somewhat unique, displaying both electrical conductivity and photo-
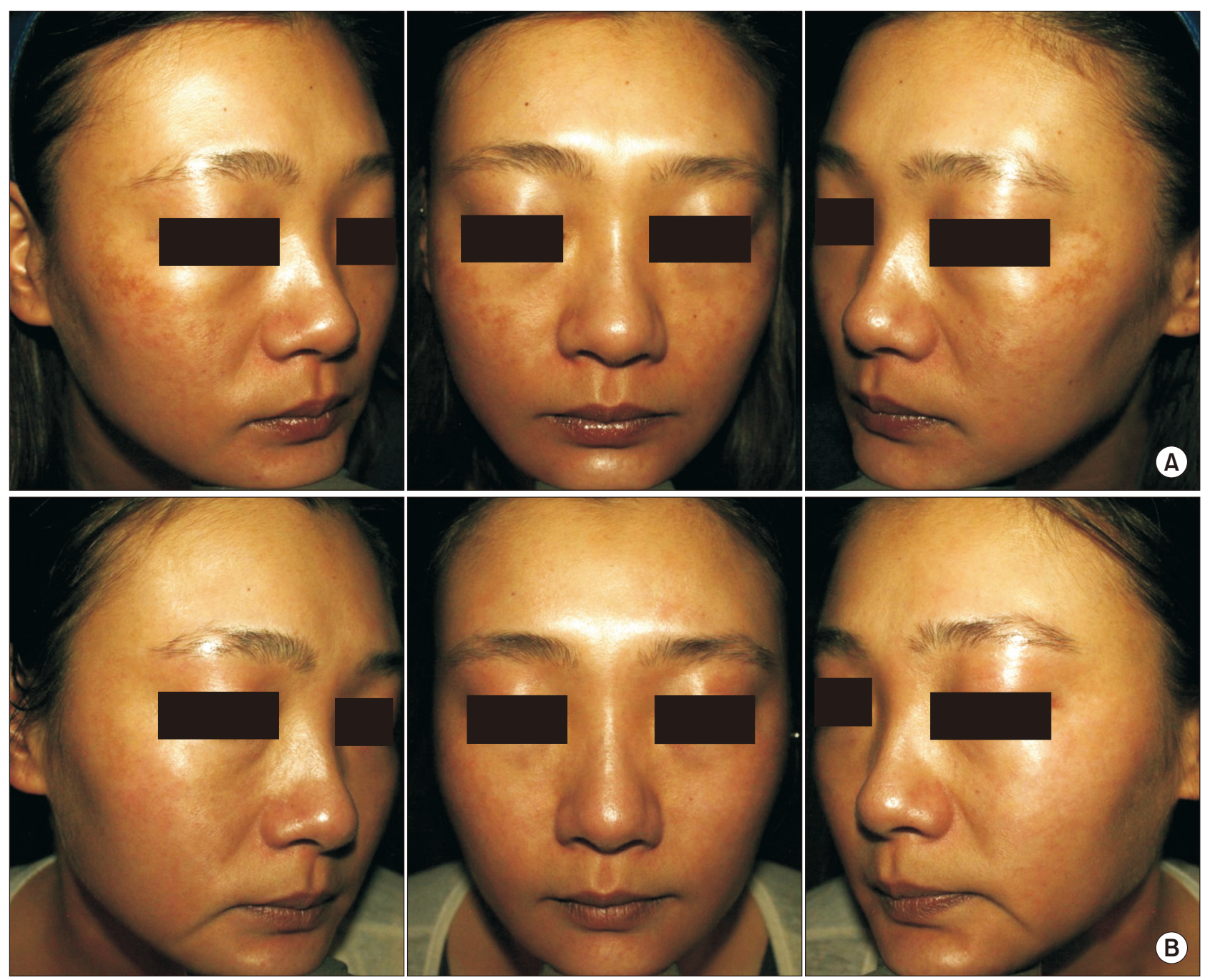

Fig. 3. Representative photographs of a 37-year-old female patient with melasma. (A) Baseline and (B) one month after six sessions of combined IBPRF and laser toning treatment. 

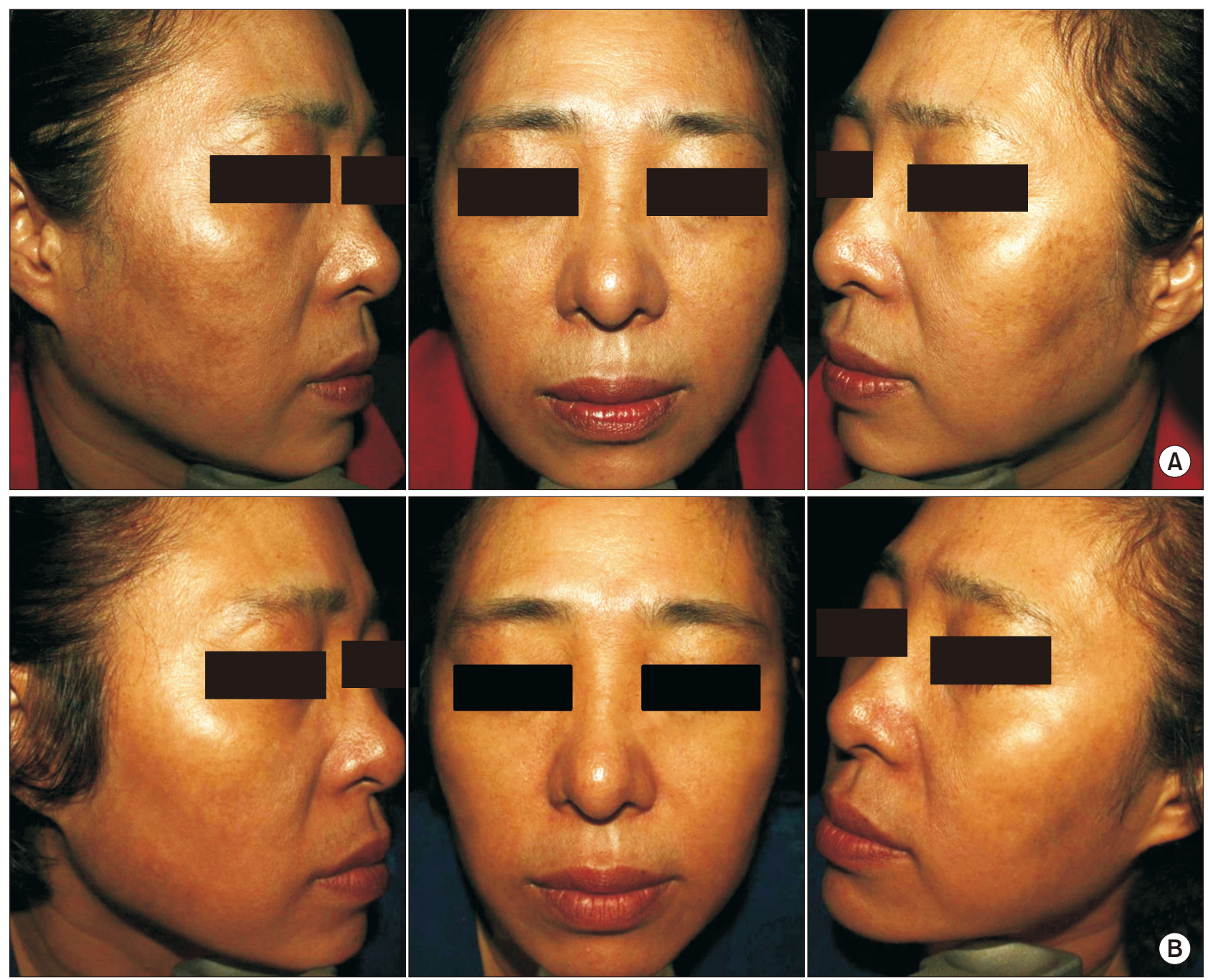

Fig. 4. Representative photographs of a 56-year-old female patient with melasma and rebound hyperpigmentation. (A) Baseline and (B) one month after eight sessions of IBPRF monotherapy.

conductivity. Eumelanin, one of three basic forms of melanin, shows pronounced electrical conductivity, both dark and photo induced. Under certain humidity, temperature, and electric field conditions, solid eumelanin pellets exhibit threshold and memory switching. ${ }^{12}$ The electrical properties (light, dark, DC, and AC) of eumelanin appear to be dominated by the hydration state of the tissue, in which a large range of $\mathrm{DC}$ electrical conductivities are reported $\left(10^{-15}-10^{-13} \mathrm{~S} / \mathrm{cm}\right) .{ }^{12}$ Based upon a typical Mott-Davies model, activation energies have been calculated from temperature dependent conductivity measurements, from which a wide range of values are reported (c. 0.5 $3.4 \mathrm{eV})$. Meanwhile, however, de Albuquerque et al. (2005, 2006) recently reported a value of $1.7 \mathrm{eV}$ in synthetic eumelanin films using photo-pyro-electric spectroscopy. ${ }^{13}$

In addition to the above, RF treatment also appears to stimulate healing of the basement membrane (BM). Indeed, electrical current has been found to help induce wound healing. ${ }^{14,15}$ Recently, pendulous melanocytes have drawn greater interest in melasma treatment. High energy laser treatment targets the BM, after which pendulous melanocytes shift downward into the dermis. ${ }^{16}$ We suggest that RF irradiation thickens and heals damaged BMs for improvement in $\mathrm{PIH}$.

Alternating current focuses along the outer surfaces of conductors. This phenomenon is called the "skin effect". ${ }^{17}$ This effect is beneficial to melasma treatment and preventing $\mathrm{PIH}$. For example, for fine vessels in the reticular dermis, if the resistance of the reticular dermis is 400 mohm and the intravascular resistance is $0 \mathrm{mohm}$, alternating current will accumulate on the vascular surface, since vessels behave as conductors (Fig. 5). Thus, pulsed 
Electrical current

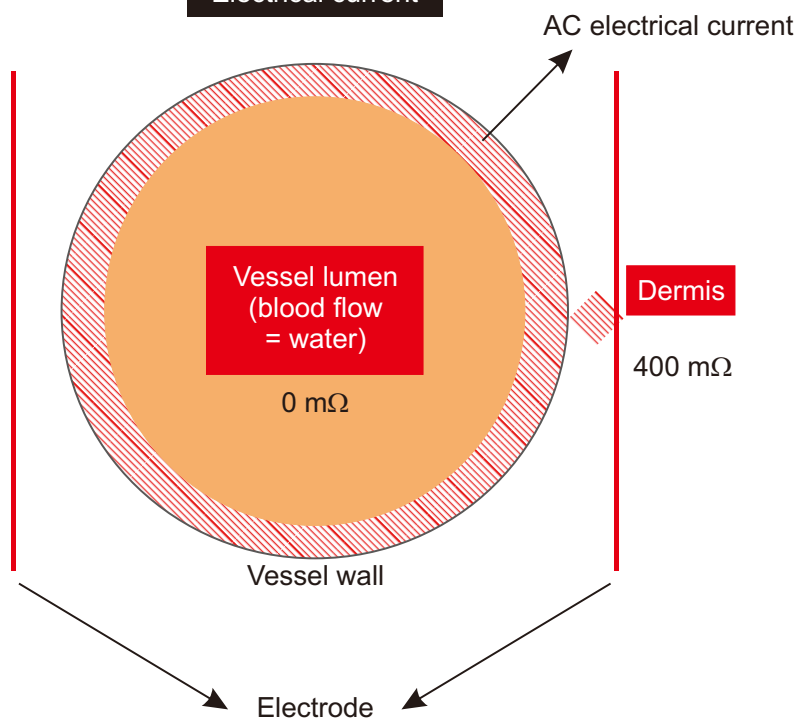

Fig. 5. Electrical current accumulates on the surface of the conductor (vessel wall), known as the "skin effect."

RF targets the vascular surface, and then, selective vessels with a certain diameter are destroyed. Large vessels remain intact due to the "sink effect," which is more important than the skin effect: the sink effect refers to moving fluid (blood) that takes away heat energy from the conductor surface. Thus, the selectivity of RF treatment for vessel diameter can be applied to melasma or vascular treatment to improve diffuse erythema after IBPRF treatment. ${ }^{17}$

The dermal environment is also improved after RF treatment. We suggest that, first, RF accelerates washing out of dermal melanin, followed by stimulation of neocollagenesis. ${ }^{18}$ This improved dermal environment helps boost melasma and PIH treatment results. Nevertheless, our propositions should be verified in biopsied results, and the safety range of pulsed RF should be confirmed.

In conclusion, no severe complications related to IBPRF treatment were recorded in the present study, and patients reported satisfaction in the appearance of their lesions after seven to eight treatment sessions. Our experiences suggest that IBPRF is effective and safe for treating melasma and $\mathrm{RH}$.

\section{ACKNOWLEDGEMENTS}

We would like to thank Anthony Thomas Milliken, ELS (Editing Synthase, Seoul, Korea) for his help with the editing of this manuscript.

\section{REFERENCES}

1. Kang HY, Ortonne JP. What should be considered in treatment of melasma. Ann Dermatol 2010;22:373-8.

2. Ortonne JP, Passeron T. Melanin pigmentary disorders: treatment update. Dermatol Clin 2005;23:209-26.

3. Negishi K, Kushikata N, Tezuka Y, Takeuchi K, Miyamoto E, Wakamatsu S. Study of the incidence and nature of "very subtle epidermal melasma" in relation to intense pulsed light treatment. Dermatol Surg 2004;30:881-6; discussion 886.

4. Lee HS, Won CH, Lee DH, An JS, Chang HW, Lee JH, et al. Treatment of melasma in Asian skin using a fractional 1,550nm laser: an open clinical study. Dermatol Surg 2009;35:1499504.

5. Jeong SY, Chang SE, Bak H, Choi JH, Kim IH. New melasma treatment by collimated low fluence $\mathrm{Q}$-switched Nd:YAG laser. Korean J Dermatol 2008;46:1163-70.

6. Polnikorn N. Treatment of refractory dermal melasma with the MedLite C6 Q-switched Nd:YAG laser: two case reports. J Cosmet Laser Ther 2008;10:167-73.

7. Cho SB, Kim JS, Kim MJ. Melasma treatment in Korean women using a 1064-nm Q-switched Nd:YAG laser with low pulse energy. Clin Exp Dermatol 2009;34:e847-50.

8. Kim MJ, Kim JS, Cho SB. Punctate leucoderma after melasma treatment using 1064-nm Q-switched Nd:YAG laser with low pulse energy. J Eur Acad Dermatol Venereol 2009;23:960-2.

9. Elsner P, Barel AO, Berardesca E, Gabard B, Serup J. Skin bioengineering techniques and applications in dermatology and cosmetology. Basel: Karger; 1998. p. 38-47.

10. Na J, Zheng Z, Dannaker C, Lee SE, Kang JS, Cho SB. Electromagnetic initiation and propagation of bipolar radiofrequency tissue reactions via invasive non-insulated microneedle electrodes. Sci Rep 2015;5:16735.

11. Sadick NS, Makino Y. Selective electro-thermolysis in aesthetic medicine: a review. Lasers Surg Med 2004;34:91-7.

12. Guo L, Kubat NJ, Isenberg RA. Pulsed radio frequency energy (PRFE) use in human medical applications. Electromagn Biol Med 2011;30:21-45.

13. Meredith P, Sarna T. The physical and chemical properties of eumelanin. Pigment Cell Res 2006;19:572-94.

14. Nuccitelli R, Nuccitelli P, Li C, Narsing S, Pariser DM, Lui K. The electric field near human skin wounds declines with age and provides a noninvasive indicator of wound healing. Wound Repair Regen 2011;19:645-55.

15. Zhao M, Penninger J, Isseroff RR. Electrical activation of wound-healing pathways. Adv Skin Wound Care 2010;1:56773.

16. Lee DJ, Park KC, Ortonne JP, Kang HY. Pendulous melanocytes: a characteristic feature of melasma and how it may occur. Br J Dermatol 2012;166:684-6. 
Invasive Pulsed Bipolar Radiofrequency Hyoung Moon Kim and Min Ji Lee

17. Kim EH, Kim YC, Lee ES, Kang HY. The vascular characteristics of melasma. J Dermatol Sci 2007;46:111-6.

18. Hantash BM, Ubeid AA, Chang H, Kafi R, Renton B. Bipolar fractional radiofrequency treatment induces neoelastogenesis and neocollagenesis. Lasers Surg Med 2009;41:1-9. 